\title{
Proton decay and light sterile neutrinos
}

\author{
Juan C. Helo, ${ }^{a, b}$ Martin Hirsch ${ }^{c}$ and Toshihiko Ota ${ }^{d}$ \\ ${ }^{a}$ Departamento de Física, Facultad de Ciencias, Universidad de La Serena, \\ Avenida Cisternas 1200, La Serena, Chile \\ ${ }^{b}$ Centro-Científico-Tecnológico de Valparaíso, \\ Casilla 110-V, Valparaíso, Chile \\ ${ }^{c}$ AHEP Group, Instituto de Física Corpuscular, \\ C.S.I.C./Universitat de València Edificio de Institutos, \\ de Paterna, Apartado 22085, E-46071 València, Spain \\ ${ }^{d}$ Department of Physics, Yachay Tech, Hacienda San José s/n y Proyecto Yachay, \\ 100115 San Miguel de Urcuquí, Ecuador \\ E-mail: jchelo@userena.cl, mahirsch@ific.uv.es, tota@yachaytech.edu.ec
}

ABSTRACT: Within the standard model, non-renormalizable operators at dimension six $(d=6)$ violate baryon and lepton number by one unit and thus lead to proton decay. Here, we point out that the proton decay mode with a charged pion and missing energy can be a characteristic signature of $d=6$ operators containing a light sterile neutrino, if it is not accompanied by the standard $\pi^{0} e^{+}$final state. We discuss this effect first at the level of effective operators and then provide a concrete model with new physics at the TeV scale, in which the lightness of the active neutrinos and the stability of the proton are related.

Keywords: Beyond Standard Model, Neutrino Physics

ARXIV EPRINT: 1803.00035 


\section{Contents}

1 Introduction 1

2 Effective operators and proton decay modes $\quad 2$

3 Longevity of proton and lightness of neutrino 5

$\begin{array}{llr}4 & \text { Conclusions } & 9\end{array}$

\section{Introduction}

The observed baryon asymmetry of the universe (BAU) requires that baryon number is violated at high energy scales. In the standard model (SM), $B+L$ is violated by nonperturbative effects, such as instantons [1,2] or the sphaleron [3]. However, as is wellknown, the SM cannot explain the observed value of the BAU [4]. Beyond these nonperturbative effects, one can also write down $B$ and $L$ violating operators at the nonrenormalizable level, as has been discussed already nearly 40 years ago [5-7]. Consequently, many ultra-violet completions of the SM contain $B$ and/or $L$ violating interactions also at the renormalizable level, the prime example being Grand Unified Theories (GUTs). In particular, $d=6$ operators lead to proton decay, but searches for proton decay so far have yielded only lower bounds, in the range of $\left(10^{32}-10^{34}\right)$ yrs, depending on the final state [8-10]. Usually these negative results are interpreted as a lower limit on the energy scale of some GUT.

Neutrino masses are much smaller than all other fermion masses. It is often argued that this smallness could be understood if neutrinos are Majorana particles; for a recent review on theoretical aspects of neutrino masses, see e.g., ref. [11]. However, we have not observed any lepton number violating (LNV) process so far and limits on neutrinoless double beta decay $(0 \nu \beta \beta)$ for example have reached now $10^{26}$ yrs $[12,13]$. Thus neutrinos could still be Dirac particles. Although much less known than the Majorana case, the study of small Dirac neutrino masses has actually quite a long history [14-20]. Interest in Dirac neutrino masses has been renewed recently [21-34], in particular its possible connection with (cold) dark matter.

In this paper, we ask the question: could the smallness of the neutrino mass and the longevity of the proton be related? In other words, can the mechanism that suppresses proton decay operators also suppress neutrino masses? First, we study this question at the level of effective operators. We point out that the decay mode $p \rightarrow \pi^{+}+$missing energy $\left(\pi^{+}+\not E\right)$ with absence of $p \rightarrow \pi^{0} \ell^{+}$is a characteristic signature of effective $d=6$ operators with a light SM singlet fermion, aka "sterile" neutrino. This singlet fermion could be the Dirac partner of the ordinary neutrinos. Next, we discuss this in a simple model in which 
both neutrino masses and proton decay share a common origin. In the model, $B-L$ is conserved, thus neutrinos are Dirac particles. Their masses arise at the 1-loop level and, as in the model of ref. [22], the particles generating the loop are candidates for the dark matter. Proton decay arises also at the 1-loop level and shares interactions and particles with the loop diagram for neutrino masses, therefore the smallness of neutrino mass is directly related to the longevity of the proton. We also consider different experimental constraints on the model parameters from neutrino masses, proton decay and searches for lepton flavour violation, and briefly discuss possible LHC signals of the model.

Before closing this section, we mention that a similar idea, relating neutrinos and proton decay, has been discussed with a particular model recently in ref. [35]. However, differing from our setup, in ref. [35] neutrinos are Majorana particles, thus $0 \nu \beta \beta$ decay should exist in their case. We also refer to ref. [36], where possible relations between neutrino mass and proton decay are discussed at the level of higher dimensional effective operators in the context of GUTs, leading to Majorana neutrinos.

The rest of this paper is organized as follows. In the next section we discuss $d=6$ operators and proton decay modes with a light sterile neutrino. In section 3 we present a concrete model, which generates $d=6$ proton decay with $\pi^{+}+\not E$ and (Dirac) neutrino masses at 1-loop and discuss its phenomenology. We then close the paper with a short summary.

\section{Effective operators and proton decay modes}

At $d=6$, the baryon-number-violating operators which are invariant under the SM gauge symmetries can be written as $[5-7]:^{1}$

$$
\begin{aligned}
\mathcal{O}_{1} & =\left[\overline{d_{R}} u_{R}\right]\left[\overline{Q^{c}} L\right], \\
\mathcal{O}_{2} & =\left[\overline{Q^{c}} Q\right]\left[\overline{u_{R}^{c}} e_{R}\right], \\
\mathcal{O}_{3} & =\left[\overline{Q^{c}} Q\right]_{1}\left[\overline{Q^{c}} L\right]_{1}, \\
\mathcal{O}_{4} & =\left[\overline{Q^{c}} Q\right]_{3}\left[\overline{Q^{c}} L\right]_{3}, \\
\mathcal{O}_{5} & =\left[\overline{d_{R}} u_{R}\right]\left[\overline{u_{R}^{c}} e_{R}\right],
\end{aligned}
$$

where for simplicity we have suppressed all flavour and colour indices. The subscripts 1 and 3 at the brackets represent the singlet and the triplet combinations of $\mathrm{SU}(2)_{L}$. Operators with the same fields but Lorentz structures different from $\mathcal{O}_{1-5}$ can be rewritten as combinations of these basis operators via Fierz transformations. For example,

$$
\left[\overline{Q^{c}}\left(\sigma^{\rho}\right) u_{R}\right]\left[\overline{d_{R}^{c}}\left(\bar{\sigma}_{\rho}\right) L\right]=2 \mathcal{O}_{1}
$$

All effective operators listed above respect $B-L$, but have $\Delta(B+L)=2$.

Extending the particle content of the SM by singlet fermion fields $N$ with one unit of lepton number, one can write down the following two additional operators, which are both

\footnotetext{
${ }^{1}$ Effective operators, which lead to purely leptonic decay modes of the proton, appear only at $d=9$, see refs. [37, 38]. For proton decay operators with mass dimensions higher than $d=6$, see also refs. [36, 39-42].
} 


\begin{tabular}{|cccc|}
\hline Modes $(p)$ & $\pi^{+}+\not E$ & $\pi^{0} e^{+}$ & $K^{+}+\not$ \\
\hline Current [yrs] & $3.9 \cdot 10^{32}[8]$ & $1.6 \cdot 10^{34}[9]$ & $5.9 \cdot 10^{33}[10]$ \\
Future [yrs] & & $1.2 \cdot 10^{35}[48]$ & $>3 \cdot 10^{34}[49]$ \\
\hline $\mathcal{O}_{1}$ & $\checkmark$ & $\checkmark$ & $\checkmark$ \\
$\mathcal{O}_{2}$ & - & $\checkmark$ & - \\
$\mathcal{O}_{3}$ & $\checkmark$ & $\checkmark$ & $\checkmark$ \\
$\mathcal{O}_{4}$ & - & - & $\checkmark$ \\
$\mathcal{O}_{5}$ & - & $\checkmark$ & - \\
\hline $\mathcal{O}_{N 1}$ & $\checkmark$ & - & $\checkmark$ \\
$\mathcal{O}_{N 2}$ & $\checkmark$ & - & $\checkmark$ \\
\hline
\end{tabular}

Table 1. Operators and proton decay modes. The numbers in the "Current" and the "Future" rows are the current bounds and the future sensitivities at $90 \%$ C.L. Only the operators $\mathcal{O}_{N 1}$ and $\mathcal{O}_{N 2}$ generate $\pi^{+}+\not E$ (missing energy), without producing the decay $\pi^{0} e^{+}$.

invariant under SM gauge transformations and $B-L$, see e.g., refs. $[43-46]^{2}$

$$
\begin{aligned}
\mathcal{O}_{N 1} & =\left[\overline{Q^{c}} Q\right]\left[\overline{d_{R}^{c}} N\right], \\
\mathcal{O}_{N 2} & =\left[\overline{u_{R}^{c}} d_{R}\right]\left[\overline{d_{R}^{c}} N\right] .
\end{aligned}
$$

Proton decay final states differ depending on the operator under consideration. Operators and the corresponding decay modes are summarized in table 1, together with the current bounds and future sensitivities [8-10, 48, 49]. The final state with a charged pion and missing energy can be generated by $\mathcal{O}_{1,3}$ and $\mathcal{O}_{N 1, N 2}$. For the case of $\mathcal{O}_{1,3}$ the final state $\pi^{+}+\not E$ is caused by the emission of a left-handed neutrino. Isospin symmetry then tells us that $\mathcal{O}_{1,3}$ also generate the process with the corresponding left-handed charged lepton, which is $p \rightarrow \pi^{0} \ell^{+}$. The decay rates of the two $\mathrm{SU}(2)_{L}$-related processes are expected to fulfill the following ratio (cf. e.g., refs. [50, 51]):

$$
\Gamma\left(p \stackrel{\mathcal{O}_{1,3}}{\longrightarrow} \pi^{+} \bar{\nu}_{e}\right)=2 \Gamma\left(p \stackrel{\mathcal{O}_{1,3}}{\longrightarrow} \pi^{0} e^{+}\right) .
$$

Conversely, the operators $\mathcal{O}_{N 1, N 2}$ do not have a charged lepton counter part and thus cannot generate the decay mode with a neutral pion and a charged lepton. It seems natural then to suppose that the discovery of proton decay with final state $p \rightarrow \pi^{+}+\not{E}$ with simultaneous absence of the $\pi^{0} \ell^{+}$mode suggests that the process is caused by an operator containing a SM singlet fermion $N$. Since the decay mode $p \rightarrow \pi^{0} e^{+}$is more strongly constrained than $p \rightarrow \pi^{+}+\not \mathcal{E}$, a discovery of the $\pi^{+}+$missing mode in the next round of proton decay searches would therefore hint at the existence of a light sterile neutrino with mass below $m_{p}-m_{\pi}$.

The effective operators $\mathcal{O}_{N 1, N 2}$ with a sterile neutrino also generate neutron decay process with $\pi^{0}+\not$. One expects them to follow a particular ratio:

$$
\Gamma\left(p \stackrel{\mathcal{O}_{N 1, N 2}}{\longrightarrow} \pi^{+} \bar{N}\right)=2 \Gamma\left(n \stackrel{\mathcal{O}_{N 1, N 2}}{\longrightarrow} \pi^{0} \bar{N}\right) .
$$

\footnotetext{
${ }^{2}$ Nucleon decays with the operator $\mathcal{O}_{N 2}$ are discussed in ref. [47] in relation with dark matter.
} 


\begin{tabular}{|ccc|}
\hline Modes $(n)$ & $\pi^{0}+\not \mathbf{r}$ & $\pi^{-} e^{+}$ \\
\hline Current [yrs] & $1.1 \cdot 10^{33}[8]$ & $5.3 \cdot 10^{33}[9]$ \\
Future [yrs] & & $3.8 \cdot 10^{34}[48]$ \\
\hline $\mathcal{O}_{1}$ & $\checkmark$ & $\checkmark$ \\
$\mathcal{O}_{2}$ & - & $\checkmark$ \\
$\mathcal{O}_{3}$ & $\checkmark$ & $\checkmark$ \\
$\mathcal{O}_{4}$ & - & - \\
$\mathcal{O}_{5}$ & - & $\checkmark$ \\
\hline $\mathcal{O}_{N 1}$ & $\checkmark$ & - \\
$\mathcal{O}_{N 2}$ & $\checkmark$ & - \\
\hline
\end{tabular}

Table 2. Same as table 1 but for neutron decay modes. Here again, the discovery of $\pi^{0}+\not E$ with simultaneous absence of $\pi^{-} \ell^{+}$suggests the existence of an effective operator containing a light sterile neutrino.

The operators and the corresponding neutron decay modes are listed in table 2, and the current bounds and the future sensitivities are found in refs. [8, 9, 48]. Again, for $\mathcal{O}_{N 1, N 2}$ there are no decays to charged leptons, $\pi^{-} e^{+}$, thus the same logic holds also for neutron decays and observation of $\pi^{0}+\not$ can be interpreted as a hint for a light sterile state.

At this point, we have to add a word of caution to the above discussion. The simple arguments presented are based on $\mathrm{SU}(2)_{L}$ invariance, used in the construction of all nonrenormalizable operators. While certainly $\mathrm{SU}(2)_{L}$ is restored at high energies, and thus, all ultra-violet completions of the SM should respect it, this by no means implies that $\mathrm{SU}(2)_{L}$ breaking effects are guaranteed to be negligible. We will discuss briefly two particular examples for setups with possibly sizable $\mathrm{SU}(2)_{L}$ violating effects.

The study of proton decay in supersymmetric (SUSY) GUTs has a long history, see e.g., refs. [52-61]. In SUSY-GUT frameworks, the leading contributions to proton decay come usually from one-loop diagrams which contain $B$ and $L$ violating dimension-five operators with two sfermions and the so called "dressing" of the operators with a gaugino or a higgsino, which converts the sfermions to the corresponding fermions. The flavour structure of the Yukawa interactions entering in this "dressing" diagram can lead to a large difference between the rate of the decay $p \rightarrow \pi^{+} \bar{\nu}$ and that of $p \rightarrow \pi^{0} \ell^{+}$. In fact, the $\pi^{+} \bar{\nu}$ decay can become more important than the $\pi^{0} \ell^{+}$mode in a large class of the SUSY-GUT models, see for example ref. [56]. However, the dominant proton decay mode in SUSY-GUTs is in general $p \rightarrow K^{+} \bar{\nu}$. Therefore, the discovery of the $p \rightarrow \pi^{+}+\not E$

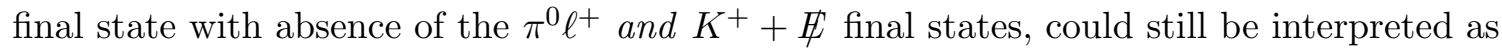
a hint for a light sterile state even in supersymmetric frameworks. A notable exception from this argument is, however, the SUSY SO(10) model discussed in ref. [62]. Here, the $p \rightarrow \pi^{+} \bar{\nu}$ may become the dominant mode in part of the parameter space, in which the decay $p \rightarrow K^{+} \bar{\nu}$ is minimized in order to obey the experimental bounds.

As the second example for the $\mathrm{SU}(2)_{L}$ violation effect, we mention the model of ref. [35]. Here, proton decay is generated by a $d=7$ operator $\left(u_{X} d_{X}\right) d_{R}\left(H^{0 *} \bar{\nu}-H^{-} l^{+}\right)$, where $X=L$ or $X=R$ and $H$ stands for the SM Higgs field. ${ }^{3}$ The vacuum expectation value of the

\footnotetext{
${ }^{3}$ This $d=7$ operator violates $B-L$ as is demonstrated in ref. [63].
} 


\begin{tabular}{|ccc|}
\hline$\left(\mathrm{SU}(3)_{c}, \mathrm{SU}(2)_{L}, \mathrm{U}(1)_{Y}, \mathrm{U}(1)_{B-L}\right)$ & $Z_{2}^{(A)}$ & $Z_{2}^{(B)}$ \\
\hline$L(\mathbf{1}, \mathbf{2},-1 / 2,-1)$ & + & - \\
$e_{R}(\mathbf{1}, \mathbf{1},-1,-1)$ & + & - \\
Other SM particles & + & + \\
\hline$N(\mathbf{1}, \mathbf{1}, 0,-1)$ & - & + \\
\hline$\eta(\mathbf{1}, \mathbf{2},+1 / 2,0)$ & - & - \\
$S^{\prime}(\mathbf{1}, \mathbf{1}, 0,0)$ & - & + \\
$\psi^{\prime}=\left(\psi_{L}^{\prime}, \psi_{R}^{\prime}\right)(\mathbf{1}, \mathbf{1}, 0,-1)$ & $(+,-)$ & $(+,+)$ \\
$S(\mathbf{3}, \mathbf{1},-1 / 3,-2 / 3)$ & - & + \\
$\psi=\left(\psi_{L}, \psi_{R}\right)(\overline{\mathbf{3}}, \mathbf{2},-1 / 6,-1 / 3)$ & $(-,-)$ & $(+,+)$ \\
\hline
\end{tabular}

Table 3. Particle content of the model, together with the corresponding charge assignments. $Z_{2}^{(A)}$ is broken only by the mass term of the fermion field $\psi^{\prime} . Z_{2}^{(B)}$ is broken softly via the $\mu$-term, see eq. (3.2).

Higgs field picks out the neutrino term exclusively from the effective operator. The $\pi^{0} \ell^{+}$ mode can also be generated from the $d=7$ operator, but it is suppressed relative to the $\pi^{+}+\not E$ mode, because it requires an extra $W$ insertion. Thus, with $d=7$ operators (and correspondingly higher dimensional ones) involving Higgs fields, $\mathrm{SU}(2)_{L}$ violation occurs naturally, restricting our argument to $d=6$ operators.

In short, we have pointed out that (in the absence of any experimental indication for $\mathrm{TeV}$-scale supersymmetry) the combination of different proton decay final states can provide hints for (or against) the existence of light sterile neutrino states. This argument is based on the assumption of (at least approximate) $\mathrm{SU}(2)_{L}$ invariance. We note that all $d=6$ operators conserve $B-L$, thus these sterile states could be the Dirac partners of the ordinary neutrinos. In the next section, we will discuss the relation between the stability of proton and the lightness of the neutrino in a concrete model with TeV-scale new physics (NP).

\section{Longevity of proton and lightness of neutrino}

Here we discuss how a possible relation between the smallness of neutrino masses and the stability of the proton can arise in a concrete model. The particle content of this model is given in table 3 .

The model is described by the following Lagrangian: ${ }^{4}$

$$
\mathscr{L}=\mathscr{L}_{1}+\mathscr{L}_{2}
$$

where

$$
\begin{aligned}
\mathscr{L}_{1}= & \left(Y_{1}\right)^{\alpha}\left(\overline{Q^{c}}{ }_{\alpha}\right)\left(\psi_{R}^{c}\right) S+\left(Y_{2}\right)^{\alpha}\left(\overline{\psi_{L}{ }^{c}}\right)\left(Q_{\alpha}\right) S^{\prime \dagger}+\left(Y_{3}\right)^{\alpha}\left(\overline{\psi_{L}^{\prime}}\right)\left(N_{\alpha}\right) S^{\prime}+\left(Y_{4}\right)^{\alpha}\left(\overline{d_{R}{ }^{c}}{ }_{\alpha}\right)\left(\psi_{R}^{\prime}\right) S^{\dagger} \\
& +\left(Y_{\nu}\right)^{\alpha}\left(\overline{\psi_{R}^{\prime}}\right)\left(L_{\alpha}\right) \eta-M_{\psi}\left(\overline{\psi_{L}}\right)\left(\psi_{R}\right)-M_{\psi^{\prime}}\left(\overline{\psi_{L}^{\prime}}\right)\left(\psi_{R}^{\prime}\right) \\
& +\mu \eta^{\dagger} H S^{\prime}+\text { H.c. }-M_{S}^{2} S^{\dagger} S-M_{S^{\prime}}^{2} S^{\prime \dagger} S^{\prime}
\end{aligned}
$$

\footnotetext{
${ }^{4}$ For a complex scalar $S^{\prime}$, one can write also the terms $\left(Y_{2}^{\prime}\right)^{\alpha}\left(\overline{\psi_{L}{ }^{c}}\right)\left(Q_{\alpha}\right) S^{\prime}$ and $\left(Y_{3}^{\prime}\right)^{\alpha}\left(\overline{\psi_{L}^{\prime}}\right)\left(N_{\alpha}\right) S^{\prime \dagger}$. Since these do not lead to any new phenomenology we have suppressed them for brevity.
} 

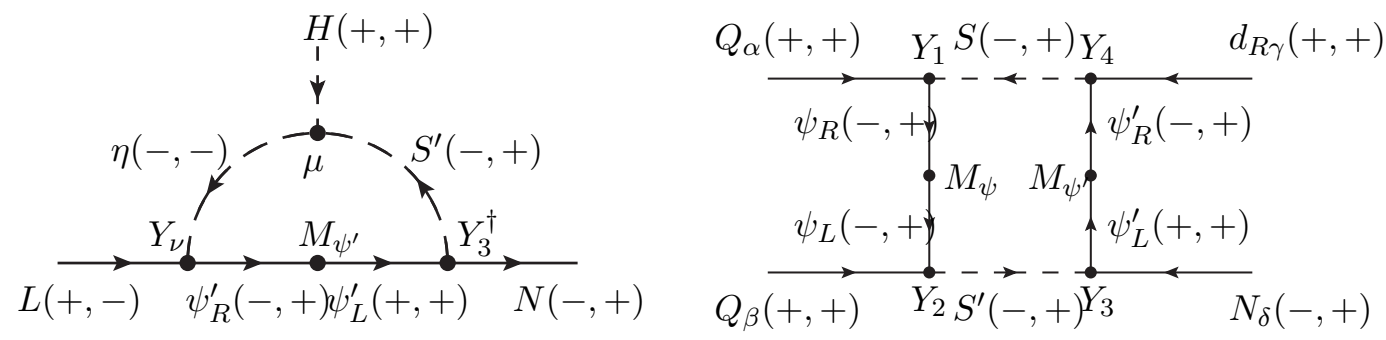

Figure 1. Diagrams for the Dirac neutrino mass and the $d=6$ proton decay operator. The signs $+/$ - represent the charges under the $\left(Z_{2}^{(A)}, Z_{2}^{(B)}\right)$ symmetries. $Z_{2}^{(A)}$ is broken only via the mass term $M_{\psi^{\prime}}$ of the fermion field $\psi^{\prime}$, and $Z_{2}^{(B)}$ is softly broken with the trilinear $\mu$ term. See text.

and

$$
\mathscr{L}_{2}=\left(Y_{4}^{\prime}\right)^{\alpha}\left(\overline{d_{R}^{c}}{ }_{\alpha}\right) N S^{\dagger}+\left(Y_{N}\right)_{\alpha}{ }^{\beta} \bar{N}^{\alpha} L_{\beta} \eta-M_{\psi^{\prime} N} \overline{\psi_{L}^{\prime}} N+\text { H.c.. }
$$

The terms in eq. (3.2) are needed for the consistency of the model, while the terms in eq. (3.3) are optional, see below. Here the parameter $\mu$ has dimension of mass. We have assumed that there are three copies of $N$, but only one copy of the fields $\psi_{L}, \psi_{R}, \psi_{L}^{\prime}$ and $\psi_{R}^{\prime}$, for simplicity. Note that at least two copies of $N$ are needed, since neutrino oscillation data require two non-zero mass eigenstates for the active neutrinos.

The transformation properties/charges of all new particles are listed in table 3. Both, $\eta$ and $S^{\prime}$ have to be inert scalars, i.e. $\left\langle\eta^{0}\right\rangle \equiv 0 \equiv\left\langle S^{\prime}\right\rangle$. Two additional $Z_{2}$ symmetries are imposed: since the Dirac neutrino mass term and the proton decay operator $\mathcal{O}_{N 1}$ violate the $Z_{2}^{(A)}$ symmetry, they can appear only in connection with the mass term of $\psi^{\prime}$, which guarantees that they are radiatively generated. The loop diagrams for the proton decay operator and the Dirac neutrino mass term are shown in figure 1 . The symmetry $Z_{2}^{(B)}$ is necessary to eliminate unwanted terms in the Lagrangian that could generate the standard proton decay operators, see eqs. $(2.1)-(2.5)$. Note that, since $Z_{2}^{(B)}$ is broken softly by the $\mu$-term, the proton decay operators $\mathcal{O}_{1}-\mathcal{O}_{5}$ will appear at higher loop order or as higher dimensional diagrams. We have found that for these operators the lowest order contribution comes from a 1-loop $d=8$ diagram which gives only sub-dominant effects in comparison with the diagram shown in figure 1.

In addition to the neutrino mass diagram on the left of figure 1, there is also a possible diagram with $N$ and $\psi_{R}^{\prime}$ exchanged, due to the terms in eq. (3.3). The presence of these terms allows, in principle, to suppress the neutrino mass additionally by a Dirac seesaw [14]. However, these terms can equally well be eliminated by an additional $Z_{2}$ for the particles in the loops in figure 1. This option has the advantage that the model at the same time could also explain the dark matter problem, see below. We will therefore not discuss the possibility of obtaining a Dirac seesaw suppression further. The additional Yukawa interaction $Y_{4}^{\prime}$ provides another loop diagram similar to the one shown on the right side in figure 1. However, since this contribution does not make any qualitative difference in our argument, we do not discuss this interaction further in the present study.

The model described by eq. (3.2) and table 3 draws some inspiration from the scotogenic model for Dirac neutrino mass, which was presented in ref. [22]. However, the authors 
of ref. [22] discuss only Dirac neutrino masses, while our variant induces also proton decay and in fact, relates the rates of the two processes as follows. In our model the charge assignments of one of the $Z_{2}$ symmetries are modified to allow the proton decay operator. More concretely, the $Z_{2}^{(A)}$ symmetry is broken with the mass term of the new fermion field $\psi^{\prime}$ in our choice, instead of the trilinear scalar coupling $\mu$. With this assignment, both the Dirac neutrino mass and the proton decay can occur only via a $Z_{2}^{(A)}$ symmetry breaking mass term $M_{\psi^{\prime}}\left(\overline{\psi_{L}^{\prime}}\right)\left(\psi_{R}^{\prime}\right)$.

Let us give a rough estimate for the resulting proton lifetime and Dirac neutrino mass. The Yukawa interactions given in eq. (3.2) mediate the effective operator $\mathcal{O}_{N 1}$, and the coefficient can be evaluated as

$$
\mathscr{L}_{\text {eff }}=\left(Y_{1}\right)^{\alpha}\left(Y_{2}\right)^{\beta}\left(Y_{3}\right)^{\delta}\left(Y_{4}\right)^{\gamma} M_{\psi} M_{\psi^{\prime}} I_{4} \mathcal{O}_{N 1},
$$

where $I_{4}$ is the loop integral function defined as

$$
I_{4} \equiv \int \frac{\mathrm{d}^{d} k}{(2 \pi)^{d} \mathrm{i}} \frac{1}{\left[k^{2}-M_{S}^{2}\right]\left[k^{2}-M_{S^{\prime}}^{2}\right]\left[k^{2}-M_{\psi}^{2}\right]\left[k^{2}-M_{\psi^{\prime}}^{2}\right]} .
$$

In the limit of $\Lambda_{\mathrm{NP}}=M_{S}=M_{S^{\prime}}=M_{\psi}=M_{\psi^{\prime}}$ one finds:

$$
I_{4} \rightarrow \frac{1}{16 \pi^{2}} \frac{1}{6} \frac{1}{\Lambda_{\mathrm{NP}}^{4}} .
$$

The mean lifetime $\tau$ can then be estimated with the coefficient of the effective operator, which gives:

$$
\begin{aligned}
\tau & \simeq \frac{1}{\frac{m_{p}}{32 \pi}\left[1-\frac{m_{\pi^{+}}^{2}}{m_{p}^{2}}\right]^{2}\left|W_{0}\left(Y_{1}\right)^{\alpha}\left(Y_{2}\right)^{\beta}\left(Y_{3}\right)^{\delta}\left(Y_{4}\right)^{\gamma} M_{\psi} M_{\psi^{\prime}} I_{4}\right|^{2}} \\
& \simeq 5 \cdot 10^{31}[\mathrm{yrs}]\left|\frac{10^{-20}}{\left(Y_{1}\right)^{\alpha}\left(Y_{2}\right)^{\beta}\left(Y_{3}\right)^{\delta}\left(Y_{4}\right)^{\gamma}}\right|^{2}\left[\frac{\left.\frac{1}{16 \pi^{2}} \frac{1}{6} \frac{1}{M_{\psi} M_{\psi^{\prime}} I_{4}}\right]^{2}}{},\right.
\end{aligned}
$$

where we used the form factor $W_{0}$ given in ref. [64]. It turns out that the Yukawa couplings $Y_{1,2,3,4}$ should be of the order $\lesssim \mathcal{O}\left(10^{-5}\right)$ to yield a proton decay signal detectable in the next generation experiments, in case the new physics scale $\Lambda_{\mathrm{NP}}$ is of the order of say a few $\mathrm{TeV} .{ }^{5}$ Note that the experimental bound is currently $\tau>3.9 \cdot 10^{32}$ yrs.

The coupling $Y_{3}$ is shared with the one-loop diagram of the Dirac mass term for neutrinos, see figure 1. The neutrino mass from this type of diagram has been calculated many times in the literature. It can be written as:

$$
\left(m_{\nu}\right)_{\alpha}{ }^{\beta}=\frac{s c\left(Y_{\nu}^{\dagger}\right)_{\alpha}\left(Y_{3}\right)^{\beta} M_{\psi^{\prime}}}{16 \pi^{2} \sqrt{2}}\left[\frac{M_{\zeta_{1}}^{2} \ln \frac{M_{\zeta_{1}}^{2}}{M_{\psi^{\prime}}^{2}}}{M_{\zeta_{1}}^{2}-M_{\psi^{\prime}}^{2}}-\frac{M_{\zeta_{2}}^{2} \ln \frac{M_{\zeta_{2}}^{2}}{M_{\psi^{\prime}}^{2}}}{M_{\zeta_{2}}^{2}-M_{\psi^{\prime}}^{2}}\right],
$$

\footnotetext{
${ }^{5}$ We set the new physics scale $\Lambda_{\mathrm{NP}}$ to be the TeV scale to make our model testable at the LHC. However larger values of $\Lambda_{\mathrm{NP}}$ are allowed, which in turn implies larger values of the Yukawa couplings.
} 
where $s$ and $c$ are sine and cosine of the mixing angle between the neutral components of the scalar mediators $\eta$ and $S^{\prime}$ and their mass eigenstates $\zeta_{1,2}$. Assuming the magnitude of coupling $\mu$ is set to be $\Lambda_{\mathrm{NP}}$, the same as all other mass parameters, the size of the resulting Dirac neutrino mass is estimated as

$$
m_{\nu} \simeq \frac{\left\langle H^{0}\right\rangle}{16 \pi^{2}} Y_{\nu}^{\dagger} Y_{3}=\mathcal{O}(0.1)\left[\frac{Y_{\nu}^{\dagger}}{10^{-5}}\right]\left[\frac{Y_{3}}{10^{-5}}\right][\mathrm{eV}] .
$$

A more detailed fit to neutrino data could easily be done [65]. Interestingly, to have the correct size of neutrino masses and a detectable rate for proton decay, keeping $\Lambda_{\mathrm{NP}} \sim \mathrm{TeV}$, the coupling $Y_{\nu}$ should also be roughly of order $Y_{\nu} \sim \mathcal{O}\left(10^{-5}\right)$. Note that the connection between the proton decay rate and the neutrino masses is not a one-to-one correspondence. This is because their diagrams only share the Yukawa coupling $Y_{3}$ (see figure 1).

The Yukawa coupling $Y_{\nu}$ also mediates charged lepton flavour violating (cLFV) processes, such as $\ell_{\alpha} \rightarrow \ell_{\beta}+\gamma$ at the one-loop level. We estimate the decay rate with the general formulas given in ref. [66] as

$$
\Gamma\left(\ell_{\beta} \rightarrow \ell_{\alpha} \gamma\right) \simeq \frac{e^{2} m_{\mu}^{5}}{16 \pi}\left|\left(Y_{\nu}^{\dagger}\right)_{\alpha}\left(Y_{\nu}\right)^{\beta}\left[-\bar{c}+\frac{3}{2} \bar{d}\right]\right|^{2},
$$

where the loop integral $-\bar{c}+3 \bar{d} / 2$ is given as a function of $t \equiv M_{\psi^{\prime}}^{2} / M_{\eta^{+}}^{2}$;

$$
\begin{aligned}
-\bar{c}+\frac{3}{2} \bar{d} & =\frac{\mathrm{i}}{16 \pi^{2}} \frac{1}{M_{\eta^{+}}^{2}}\left[\frac{2 t^{2}+5 t-1}{12(t-1)^{3}}-\frac{t^{2} \ln t}{2(t-1)^{4}}\right] \\
& \stackrel{t \rightarrow 1}{\longrightarrow} \frac{\mathrm{i}}{16 \pi^{2}} \frac{1}{24} \frac{1}{M_{\eta^{+}}^{2}} .
\end{aligned}
$$

Using Yukawa couplings $Y_{\nu}$ of order $\mathcal{O}\left(10^{-5}\right)$, as suggested by neutrino masses (cf. eq. (3.9)), and assuming the masses of the mediators are all at the $\mathrm{TeV}$ scale, we find that the branching ratio for $\mu \rightarrow e \gamma$ is roughly

$$
\operatorname{Br}(\mu \rightarrow e \gamma)=7 \cdot 10^{-31}\left|\frac{Y_{\nu}^{\dagger} Y_{\nu}}{10^{-10}}\right|^{2}\left[\frac{3 \mathrm{TeV}}{\Lambda_{\mathrm{NP}}}\right]^{4} .
$$

This is far below current and future sensitivities [67]. In short, the correct order of neutrino masses can be reproduced, and simultaneously the size of the signature mode $p \rightarrow \pi^{+}+$missing of the light sterile neutrino can be kept at a detectable size, while satisfying constraints from the cLFV and keeping the NP scale stays at $\mathrm{TeV}$.

Finally, let us briefly mention dark matter and LHC phenomenology. As table 3 shows, the model has two neutral particles, one fermion and one scalar. Both could be the dark matter (DM) depending on which is the lightest state. However, since both of our $Z_{2}$ 's are broken softly, one would need to introduce another symmetry, to stabilize the DM candidate. The simplest possibility is another $Z_{2}$, under which all beyond SM particles - except $N$ - are odd. This symmetry also eliminates the terms in eq. (3.3). Dark matter phenomenology for these candidates has already been discussed in ref. [22]. Here 
we only note that our preferred candidate would be the neutral scalar, since the fermionic candidate requires that $Y_{3}$ is much larger than $10^{-5}$ in order to reproduce the correct relic density [22] and such large value of $Y_{3}$ would in turn require quite a large hierarchy among the Yukawa couplings.

At the LHC, the new coloured particles in our model can be pair produced through gluon-gluon fusion. Typical cross sections for the scalars can be found in ref. [68]. Cross sections for the coloured fermions should be around a factor of two larger than those for scalars (for the same mass). The typical signature of the coloured particles are jet(s) with missing energy. The coloured scalar $S$ decays into a jet $\left(d_{R}\right)$ with missing energy $\psi^{\prime}$ through the Yukawa interaction $Y_{4}$. The decay rate is roughly

$$
\Gamma\left(S \rightarrow{\overline{\psi^{\prime}}}_{L}+d_{R \alpha}\right)=\frac{3\left|\left(Y_{4}\right)^{\alpha}\right|^{2} M_{S}}{16 \pi}\left[1-\frac{M_{\psi^{\prime}}^{2}}{M_{S}^{2}}\right]^{2} .
$$

With $M_{S}=3 \mathrm{TeV}$ and $Y_{4}=10^{-5}$, the decay rate is estimated to be $2 \cdot 10^{-8} \mathrm{GeV}$ (if $M_{S} \gg M_{\psi^{\prime}}$ ), implying the decay is prompt. ${\overline{\psi^{\prime}}}_{L}$ will decay further, if it is not the lightest neutral particle. However, $\bar{\psi}_{L}{ }_{L}$ decays invisibly, thus there is no change in the LHC signature. Leptoquark searches with the jet $+\bar{\nu}$ mode at ATLAS [69] and CMS [70] provide currently lower limits on such coloured states, which are roughly of the order of $1 \mathrm{TeV}$. However, all these searches are still based on only moderate luminosity samples and significant improvements in these searches in the high luminosity run of the LHC can be expected. As an aside we note that if $Y_{4}$ is assumed to be much smaller, say as small as $\mathcal{O}\left(10^{-9}\right)$, the lifetime of $S$ becomes order of a nanosecond. The $S$ would then hadronize before decaying, leaving an ionizing track in the detector, see for example the recent paper [71] for a discussion of experimental status.

\section{Conclusions}

In this paper we have discussed a simple model that relates the longevity of the proton with the smallness of the neutrino mass. In this model, neutrinos are Dirac particles and proton decay is dominated by the final state $\pi^{+}+\not E$. Although this is only an example model, we discussed at the level of effective $d=6$ operators, that in general the observation of proton decay with the final state $\pi^{+}+\not E$, together with the non-observation of the well-known $\pi^{0} e^{+}$final state, could be interpreted in favour of the existence of a light sterile neutrino.

We plan to study the details of the phenomenology of the model given in this letter and exhaustively explore the relation between the proton decay mode $p \rightarrow \pi^{+}+$missing and Dirac neutrino mass models with the full decomposition of the proton decay operators $\mathcal{O}_{N 1, N 2}[65]$.

Finally, we would like to mention that the discussion based on the effective operators, which is given in section 2, is valid also if the light sterile neutrino is not the Dirac partner of the ordinary neutrino. Such a sterile neutrino could be an additional Majorana neutrino, if $B-L$ is violated, or come with its own Dirac partner otherwise. Therefore, a positive result of sterile neutrino searches in short baseline oscillation experiments [72, 73] would be interesting, since it opens up the possibility for $d=6$ proton decay operators to exist that exclusively produce the $\pi^{+}+\not$ mode. 


\section{Acknowledgments}

We thank Renato Fonseca for discussions on symmetries. We thank Prof. Rabindra Mohapatra for useful discussions on proton decay processes in GUT models. This work was supported by the Spanish MICINN grants FPA2017-85216-P, SEV-2014-0398 and PROMETEOII/2014/084 (Generalitat Valenciana). J.C.H. is supported by Chile grants Fondecyt No. 1161463, Conicyt PIA/ACT 1406 and Basal FB0821.

Open Access. This article is distributed under the terms of the Creative Commons Attribution License (CC-BY 4.0), which permits any use, distribution and reproduction in any medium, provided the original author(s) and source are credited.

\section{References}

[1] G. 't Hooft, Symmetry breaking through Bell-Jackiw anomalies, Phys. Rev. Lett. 37 (1976) 8 [INSPIRE].

[2] G. 't Hooft, Computation of the quantum effects due to a four-dimensional pseudoparticle, Phys. Rev. D 14 (1976) 3432 [Erratum ibid. D 18 (1978) 2199] [InSPIRE].

[3] F.R. Klinkhamer and N.S. Manton, A saddle point solution in the Weinberg-Salam theory, Phys. Rev. D 30 (1984) 2212 [INSPIRE].

[4] A. Riotto and M. Trodden, Recent progress in baryogenesis, Ann. Rev. Nucl. Part. Sci. 49 (1999) 35 [hep-ph/9901362] [INSPIRE].

[5] S. Weinberg, Baryon and lepton nonconserving processes, Phys. Rev. Lett. 43 (1979) 1566 [INSPIRE].

[6] F. Wilczek and A. Zee, Operator analysis of nucleon decay, Phys. Rev. Lett. 43 (1979) 1571 [INSPIRE].

[7] L.F. Abbott and M.B. Wise, The effective Hamiltonian for nucleon decay, Phys. Rev. D 22 (1980) 2208 [INSPIRE].

[8] Super-Kamiokande collaboration, K. Abe et al., Search for nucleon decay via $n \rightarrow \bar{\nu} \pi^{0}$ and $p \rightarrow \bar{\nu} \pi^{+}$in Super-Kamiokande, Phys. Rev. Lett. 113 (2014) 121802 [arXiv:1305.4391] [INSPIRE].

[9] Super-Kamiokande collaboration, K. Abe et al., Search for nucleon decay into charged antilepton plus meson in 0.316 megaton-years exposure of the Super-Kamiokande water Cherenkov detector, Phys. Rev. D 96 (2017) 012003 [arXiv: 1705.07221] [INSPIRE].

[10] Super-Kamiokande collaboration, K. Abe et al., Search for proton decay via $p \rightarrow \nu \nu K^{+}$ using 260 kiloton.year data of Super-Kamiokande, Phys. Rev. D 90 (2014) 072005 [arXiv: 1408.1195] [INSPIRE].

[11] Y. Cai et al., From the trees to the forest: a review of radiative neutrino mass models, Front. in Phys. 5 (2017) 63 [arXiv:1706. 08524] [INSPIRE].

[12] KamLAND-Zen collaboration, A. Gando et al., Search for Majorana neutrinos near the inverted mass hierarchy region with KamLAND-Zen, Phys. Rev. Lett. 117 (2016) 082503 [arXiv: 1605.02889] [INSPIRE]. 
[13] M. Agostini et al., Background-free search for neutrinoless double- $\beta$ decay of ${ }^{76}$ Ge with GERDA, arXiv:1703.00570 [INSPIRE].

[14] P. Roy and O.U. Shanker, Observable neutrino dirac mass and supergrand unification, Phys. Rev. Lett. 52 (1984) 713 [Erratum ibid. 52 (1984) 2190] [INSPIRE].

[15] D. Chang and R.N. Mohapatra, Small and calculable Dirac neutrino mass, Phys. Rev. Lett. 58 (1987) 1600 [INSPIRE].

[16] R.N. Mohapatra, A model for Dirac neutrino masses and mixings, Phys. Lett. B 198 (1987) 69 [INSPIRE].

[17] R.N. Mohapatra, Left-right symmetry and finite one loop Dirac neutrino mass, Phys. Lett. B 201 (1988) 517 [INSPIRE].

[18] B.S. Balakrishna and R.N. Mohapatra, Radiative fermion masses from new physics at TeV scale, Phys. Lett. B 216 (1989) 349 [InSPIRE].

[19] K.S. Babu and X.G. He, Dirac neutrino masses as two loop radiative corrections, Mod. Phys. Lett. A 4 (1989) 61 [INSPIRE].

[20] S. Rajpoot, Model with calculable Dirac neutrino masses, Phys. Rev. D 44 (1991) 225 [INSPIRE].

[21] S. Kanemura, T. Nabeshima and H. Sugiyama, Neutrino masses from loop-induced Dirac Yukawa couplings, Phys. Lett. B 703 (2011) 66 [arXiv:1106.2480] [INSPIRE].

[22] Y. Farzan and E. Ma, Dirac neutrino mass generation from dark matter, Phys. Rev. D 86 (2012) 033007 [arXiv: 1204.4890] [INSPIRE].

[23] A. Pilaftsis, Anomalous fermion mass generation at three loops, Mod. Phys. Lett. A 28 (2013) 1350083 [arXiv: 1207.0544] [INSPIRE].

[24] S. Kanemura, K. Sakurai and H. Sugiyama, Probing models of Dirac neutrino masses via the flavor structure of the mass matrix, Phys. Lett. B 758 (2016) 465 [arXiv:1603.08679] [INSPIRE].

[25] C. Bonilla, E. Ma, E. Peinado and J.W.F. Valle, Two-loop Dirac neutrino mass and WIMP dark matter, Phys. Lett. B 762 (2016) 214 [arXiv:1607.03931] [INSPIRE].

[26] D. Borah and A. Dasgupta, Common origin of neutrino mass, dark matter and Dirac leptogenesis, JCAP 12 (2016) 034 [arXiv:1608.03872] [INSPIRE].

[27] D. Borah and A. Dasgupta, Observable lepton number violation with predominantly Dirac nature of active neutrinos, JHEP 01 (2017) 072 [arXiv: 1609.04236] [INSPIRE].

[28] W. Wang and Z.-L. Han, Naturally small Dirac neutrino mass with intermediate $\mathrm{SU}(2)_{L}$ multiplet fields, arXiv:1611.03240 [INSPIRE].

[29] E. Ma and O. Popov, Pathways to naturally small Dirac neutrino masses, Phys. Lett. B 764 (2017) 142 [arXiv: 1609. 02538] [INSPIRE].

[30] W. Wang, R. Wang, Z.-L. Han and J.-Z. Han, The B - L scotogenic models for Dirac neutrino masses, Eur. Phys. J. C 77 (2017) 889 [arXiv:1705.00414] [INSPIRE].

[31] S. Kanemura, K. Sakurai and H. Sugiyama, Neutrino mass without lepton number violation, dark matter; and a strongly first-order phase transition, Phys. Rev. D 96 (2017) 095024 [arXiv: 1705.07040] [INSPIRE]. 
[32] E. Ma and U. Sarkar, Radiative Left-Right Dirac Neutrino Mass, Phys. Lett. B 776 (2018) 54 [arXiv: 1707.07698] [INSPIRE].

[33] C.-Y. Yao and G.-J. Ding, Systematic study of one-loop Dirac neutrino masses and viable dark matter candidates, Phys. Rev. D 96 (2017) 095004 [arXiv:1707.09786] [INSPIRE].

[34] S. Centelles Chuliá, R. Srivastava and J.W.F. Valle, Seesaw roadmap to neutrino mass and dark matter, Phys. Lett. B 781 (2018) 122 [arXiv:1802.05722] [INSPIRE].

[35] P.-H. Gu, E. Ma and U. Sarkar, Connecting radiative neutrino mass, neutron-antineutron oscillation, proton decay and leptogenesis through dark matter, Phys. Rev. D 94 (2016) 111701 [arXiv: 1608.02118] [INSPIRE].

[36] A. de Gouvêa, J. Herrero-Garcia and A. Kobach, Neutrino masses, grand unification and baryon number violation, Phys. Rev. D 90 (2014) 016011 [arXiv: 1404.4057] [INSPIRE].

[37] P.J. O'Donnell and U. Sarkar, Three lepton decay mode of the proton, Phys. Lett. B 316 (1993) 121 [hep-ph/9307254] [INSPIRE].

[38] T. Hambye and J. Heeck, Proton decay into charged leptons, Phys. Rev. Lett. 120 (2018) 171801 [arXiv: 1712.04871] [INSPIRE].

[39] K.S. Babu and R.N. Mohapatra, B-L violating proton decay modes and new baryogenesis scenario in SO(10), Phys. Rev. Lett. 109 (2012) 091803 [arXiv:1207.5771] [INSPIRE].

[40] L. Lehman, Extending the standard model effective field theory with the complete set of dimension-7 operators, Phys. Rev. D 90 (2014) 125023 [arXiv:1410.4193] [InSPIRE].

[41] S. Bhattacharya and J. Wudka, Dimension-seven operators in the standard model with right handed neutrinos, Phys. Rev. D 94 (2016) 055022 [arXiv: 1505. 05264] [INSPIRE].

[42] R.M. Fonseca, M. Hirsch and R. Srivastava, $\Delta L=3$ processes: proton decay and the LHC, Phys. Rev. D 97 (2018) 075026 [arXiv: 1802.04814] [INSPIRE].

[43] F. del Aguila, S. Bar-Shalom, A. Soni and J. Wudka, Heavy Majorana neutrinos in the effective lagrangian description: application to hadron colliders, Phys. Lett. B 670 (2009) 399 [arXiv: 0806. 0876] [INSPIRE].

[44] R. Alonso et al., Renormalization group evolution of dimension-six baryon number violating operators, Phys. Lett. B 734 (2014) 302 [arXiv:1405.0486] [INSPIRE].

[45] Y. Liao and X.-D. Ma, Operators up to dimension seven in standard model effective field theory extended with sterile neutrinos, Phys. Rev. D 96 (2017) 015012 [arXiv:1612.04527] [INSPIRE].

[46] L. Merlo, S. Saa and M. Sacristán-Barbero, Baryon non-invariant couplings in Higgs effective field theory, Eur. Phys. J. C 77 (2017) 185 [arXiv: 1612.04832] [InSPIRE].

[47] H. Davoudiasl, Nucleon decay into a dark sector, Phys. Rev. Lett. 114 (2015) 051802 [arXiv: 1409.4823] [INSPIRE].

[48] Hyper-Kamiokande Design Report, KEK-PREPRINT-2016-21 (2016) [ICRR-REPORT-701-2016-1].

[49] DUNE collaboration, R. Acciarri et al., Long-Baseline Neutrino Facility (LBNF) and Deep Underground Neutrino Experiment (DUNE), arXiv:1512.06148 [INSPIRE].

[50] G. Senjanović, Proton decay and grand unification, AIP Conf. Proc. 1200 (2010) 131 [arXiv:0912.5375] [INSPIRE]. 
[51] E. Golowich, Scalar mediated proton decay, Phys. Rev. D 24 (1981) 2899 [InSPIRE].

[52] P. Langacker, Grand unified theories and proton decay, Phys. Rept. 72 (1981) 185 [INSPIRE].

[53] N. Sakai and T. Yanagida, Proton decay in a class of supersymmetric grand unified models, Nucl. Phys. B 197 (1982) 533 [inSPIRE].

[54] S. Dimopoulos, S. Raby and F. Wilczek, Proton decay in supersymmetric models, Phys. Lett. B 112 (1982) 133.

[55] J.R. Ellis, D.V. Nanopoulos and S. Rudaz, GUTs 3: SUSY GUTs 2, Nucl. Phys. B 202 (1982) 43 [INSPIRE].

[56] P. Nath, A.H. Chamseddine and R.L. Arnowitt, Nucleon decay in supergravity unified theories, Phys. Rev. D 32 (1985) 2348 [inSPIRE].

[57] J. Hisano, H. Murayama and T. Yanagida, Nucleon decay in the minimal supersymmetric SU(5) grand unification, Nucl. Phys. B 402 (1993) 46 [hep-ph/9207279] [INSPIRE].

[58] V. Lucas and S. Raby, Nucleon decay in a realistic $\mathrm{SO}(10)$ SUSY GUT, Phys. Rev. D 55 (1997) 6986 [hep-ph/9610293] [INSPIRE].

[59] T. Goto and T. Nihei, Effect of RRRR dimension five operator on the proton decay in the minimal SU(5) SUGRA GUT model, Phys. Rev. D 59 (1999) 115009 [hep-ph/9808255] [INSPIRE].

[60] H. Murayama and A. Pierce, Not even decoupling can save minimal supersymmetric $\mathrm{SU}(5)$, Phys. Rev. D 65 (2002) 055009 [hep-ph/0108104] [InSPIRE].

[61] J. Hisano, D. Kobayashi, T. Kuwahara and N. Nagata, Decoupling can revive minimal supersymmetric SU(5), JHEP 07 (2013) 038 [arXiv: 1304.3651] [INSPIRE].

[62] H.S. Goh, R.N. Mohapatra, S. Nasri and S.-P. Ng, Proton decay in a minimal SUSY SO(10) model for neutrino mixings, Phys. Lett. B 587 (2004) 105 [hep-ph/0311330] [INSPIRE].

[63] A. Kobach, Baryon number, lepton number and operator dimension in the standard model, Phys. Lett. B 758 (2016) 455 [arXiv: 1604.05726] [INSPIRE].

[64] Y. Aoki, E. Shintani and A. Soni, Proton decay matrix elements on the lattice, Phys. Rev. D 89 (2014) 014505 [arXiv: 1304.7424] [INSPIRE].

[65] J.C. Helo, M. Hirsch and T. Ota, work in progress (2018).

[66] L. Lavoura, General formulae for $f_{1} \rightarrow f_{2} \gamma$, Eur. Phys. J. C 29 (2003) 191 [hep-ph/0302221] [INSPIRE].

[67] N. Atanov et al., The Mu2e crystal calorimeter, arXiv:1801.10002 [INSPIRE].

[68] I. Doršner and A. Greljo, Leptoquark toolbox for precision collider studies, JHEP 05 (2018) 126 [arXiv: 1801.07641] [INSPIRE].

[69] ATLAS collaboration, Search for scalar leptoquarks in pp collisions at $\sqrt{s}=13 \mathrm{TeV}$ with the ATLAS experiment, New J. Phys. 18 (2016) 093016 [arXiv: 1605.06035] [INSPIRE].

[70] CMS collaboration, Search for pair-production of first generation scalar leptoquarks in pp collisions at $\sqrt{s}=13 \mathrm{TeV}$ with 2.6 $\mathrm{fb}^{-1}$, CMS-PAS-EXO-16-043 (2016).

[71] T. Lenz, Searches for long-lived and highly-ionizing particles at the CMS and ATLAS experiments, PoS (LHCP2016)104 [arXiv: 1609.08369] [INSPIRE]. 
[72] LAr1-ND, ICARUS-WA104, MicroBooNE collaboration, M. Antonello et al., A proposal for a three detector short-baseline neutrino oscillation program in the Fermilab booster neutrino beam, arXiv:1503.01520 [INSPIRE].

[73] D. Cianci, A. Furmanski, G. Karagiorgi and M. Ross-Lonergan, Prospects of light sterile neutrino oscillation and CP-violation searches at the Fermilab Short Baseline Neutrino Facility, Phys. Rev. D 96 (2017) 055001 [arXiv:1702.01758] [INSPIRE]. 